INPLASY PROTOCOL

To cite: Zheng et al. The effectiveness of additional anti-reflux surgery after resection of esophagogastric junction cancer: protocol for a systematic review and metaanalysis. Inplasy protocol 2021100043. doi: 10.37766/inplasy2021.10.0043

Received: 14 October 2021

Published: 14 October 2021

Corresponding author: Hao Chen

chenhaodrs@163.com

Author Affiliation: Lanzhou University Second Hospital.

Support: Longitudinal issues.

Review Stage at time of this submission: Preliminary searches.

Conflicts of interest: None declared.

\section{The effectiveness of additional anti- reflux surgery after resection of esophagogastric junction cancer: protocol for a systematic review and meta-analysis}

Zheng, P1; Wang, N2; Yu, Y3; Gao, L4; Li, Y5; Ding, F6; Wang, B7; Chen, $\mathrm{H}^{8}$.

Review question / Objective: This study will evaluate all trials comparing the outcomes between conventional surgery and conventional surgery combined additional anti-reflux surgery aimed at investigating the effects and clinical value of additional anti-reflux surgery for esophagogastric junction cancer and to provide a reference for surgeons to design operative plans in clinical practice.

Condition being studied: Esophagogastric junction cancer.

Eligibility criteria: All randomized controlled trials (RCTs) or controlled clinical trials (methods of allocating participants to a treatment which are not strictly random, e.g., date of birth, hospital record number or alternation) published in Chinese or English will be considered for inclusion.

INPLASY registration number: This protocol was registered with the International Platform of Registered Systematic Review and Meta-Analysis Protocols (INPLASY) on 14 October 2021 and was last updated on 01 November 2021 (registration number INPLASY2021100043).

\section{INTRODUCTION}

Review question / Objective: This study will evaluate all trials comparing the outcomes between conventional surgery and conventional surgery combined additional anti-reflux surgery aimed at investigating the effects and clinical value of additional anti-reflux surgery for esophagogastric junction cancer and to provide a reference for surgeons to design operative plans in clinical practice.

Condition being studied: Esophagogastric junction cancer. 


\section{METHODS}

Participant or population: Patients with esophagogastric junction cancer diagnosed via pathological examination, and with no adjacent tissue or organ invasion, no distant metastases and suitable for radical resection will be included. There will be no restrictions regarding patients' age, sex, race, and place of residence.esophagogastric junction cancer patients.

Intervention: Radical resection of the esophagogastric junction tumor in combination with the additional anti-reflux surgeries that include fundoplication and pyloroplasty.

Comparator: Radical resection of the esophagogastric junction cancer

Study designs to be included: All randomized controlled trials (RCTs) or controlled clinical trials.

Eligibility criteria: All randomized controlled trials (RCTs) or controlled clinical trials (methods of allocating participants to a treatment which are not strictly random, e.g., date of birth, hospital record number or alternation) published in Chinese or English will be considered for inclusion.

Information sources: We will search the MEDLINE, Cochrane Library, EMBASE, CBM, CNKI, and Wanfang databases. All searches of these databases will be conducted from inception to December 2021. We will also perform a manual search in relevant professional journals and track the bibliographies of studies included in published systematic reviews or metaanalyses with a similar topic to retrieve potential additional studies.

Main outcome(s): All potential outcomes related to gastroesophageal reflux, including occurrence of reflux symptoms, reflux esophagitis, reflux occurrence based on barium meal examination, $\mathrm{pH}$ of fluid from the lower esophagus, and 24-h esophageal $\mathrm{pH}$ test will be assessed as the primary outcomes. Other outcomes, such as patients survival and post-operative complications (e.g., stenosis, infection and leak), will also been included for analysis.

Quality assessment / Risk of bias analysis: Two review authors will independently assess the risk of bias of each included trial using the Cochrane Collaboration's tool for assessing risk of bias[8]. Disagreements will be resolved by discussion between the two review authors. If a consensus could not be reached, a third review author will be employed as arbiter. The main criteria applied to measure the risk of bias include the following items: random sequence generation; allocation concealment; blinding of participants, personnel and outcome assessors; completeness of outcome data; selective reporting and other sources of bias (baseline imbalance, source of funding). The risk of bias on each criterion will be explicitly judged using the following standard: Yes (low risk of bias), No (high risk of bias), or unclear (either lack of information or uncertainty over the potential for bias).

Strategy of data synthesis: All statistical tests will be calculated using RevMan 5.3, and the magnitude of each effect will be present with $95 \%$ Cls. For dichotomous data, we used the odds ratio (OR) as a statistical index. For continuous outcomes, the mean difference (MD) will be calculated by weighing the absolute change based on group size if the trials employ comparable scales; if different scales are used to measure the same conceptual domain, we will calculate standardized mean differences (SMD) instead. Survival data will be pooled using the hazard ratios (HRs) to estimate the difference of survival between groups. Before data analysis, we will assess the heterogeneity using the $x^{2}$ and 12 tests. In addition, sources of heterogeneity will be explored using the subgroup analysis according to types of anti-reflex surgeries, pathological type, and study designs. The sensitivity analysis will be performed to test the stability of the results of meta-analysis by removing the low quality studies. The random effect model will be applied if they are determined 
to be heterogeneous ( $x 2 p<0.05,12 \geq 50 \%$ ). Instead, fixed effect model will be applied. The results will be visualized using the forest plots and pooled estimates will be reported. Statistical significance is set at $p$ $<0.05$.

Subgroup analysis: Sources of heterogeneity will be explored using the subgroup analysis according to types of anti-reflex surgeries, pathological type, and study designs.

Sensitivity analysis: The sensitivity analysis will be performed to test the stability of the results of meta-analysis by removing the low quality studies.

Country(ies) involved: China.

Keywords: esophagogastric junction cancer; additional anti-reflux surgery; protocol; systematic review; meta-analysis.

Contributions of each author:

Author 1 - Peng Zheng.

Author 2 - Na Wang.

Author 3 - Yang Yu.

Author 4 - Lei Gao.

Author 5 - Yu Li.

Author 6 - Fanghui Ding.

Author 7 - Bofang Wang.

Author 8 - Hao Chen. 